\title{
Wernicke's Encephalopathy after Pancreaticoduodenectomy: A Case Report
}

\author{
Kazuteru Monden ${ }^{a} \quad$ Hiroshi Sadamori $^{\mathrm{a}}$ Masayoshi Hioki ${ }^{\mathrm{a}}$ \\ Satoshi Ohno ${ }^{a}$ Mitsue Rikimaru ${ }^{b}$ Hiromi Saneto ${ }^{c}$ Toru Ueki ${ }^{c}$

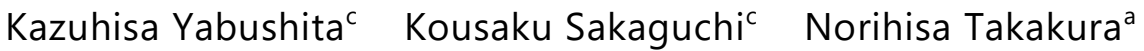 \\ aDepartment of Gastroenterological Surgery, Fukuyama City Hospital, Fukuyama, Japan; \\ ${ }^{b}$ Department of Neurology, Fukuyama City Hospital, Fukuyama, Japan; 'Department of \\ Internal Medicine, Fukuyama City Hospital, Fukuyama, Japan
}

\section{Keywords}

Wernicke's encephalopathy · Pancreaticoduodenectomy · Pancreatic fistula · Lactic acidosis

\begin{abstract}
Background: Wernicke's encephalopathy (WE) is caused by thiamine deficiency and classically appears as changes in mental status, oculomotor manifestations, and gait ataxia. WE is mostly found in patients with chronic alcoholism and malnutrition, and has rarely been reported following pancreaticoduodenectomy. Case Presentation: A 77-year-old woman was admitted to our hospital complaining of loss of appetite and weakness of the lower extremities. No abnormalities were found on blood examination. One year earlier, she had undergone pancreaticoduodenectomy for bile duct cancer, and pancreatic fistula developed as a postoperative complication. On hospital day 8 , her level of consciousness deteriorated, and she experienced difficulty bending the lower limbs and walking. Computed tomography and upper gastrointestinal series showed dilatation of the residual stomach and stenosis of the gastrojejunostomy. Laboratory test results were normal except for blood gas analysis, which revealed severe
\end{abstract}


lactic acidosis. A diagnosis of WE was suspected due to lactic acidosis secondary to thiamine deficiency. Definitive diagnosis was confirmed by magnetic resonance imaging of the brain. Intravenous infusion of high-dose thiamine (1,200 mg/day) was commenced, leading to improvement of her neurological condition. Conclusions: Physicians should recognize that pancreaticoduodenectomy, when complicated by gastrojejunostomy stenosis resulting in pancreatic fistula, can lead to WE. In this case, blood gas analysis was helpful in arriving at the correct diagnosis. A high degree of suspicion should be maintained if any of the classical features of WE are observed following pancreaticoduodenectomy.

(C) 2019 The Author(s)

Published by S. Karger AG, Basel

\section{Background}

Wernicke's encephalopathy (WE) is a neuropsychiatric syndrome resulting from a nutritional deficiency of thiamine (vitamin $\mathrm{B}_{1}$ ). The clinical triad associated with WE consists of changes in mental status, oculomotor manifestations, and gait ataxia [1]. Chronic alcoholism with malnutrition is the most common cause of WE. Hyperemesis gravidarum and parenteral nutrition without thiamine supplementation can also cause WE [1]. However, WE has not previously been recognized as a possible complication of pancreaticoduodenectomy. Early and definitive diagnosis of nonalcoholic WE is often difficult. We report the case of a patient who developed WE following pancreaticoduodenectomy. In this case, the finding of lactic acidosis on blood gas analysis was useful for the diagnosis of WE.

\section{Case Presentation}

A 77-year-old woman was referred to our hospital complaining of loss of appetite, weakness of the lower extremities, and frequent falls without any other focal neurological signs. Computed tomography (CT) of the brain showed no abnormalities. Twelve months earlier, she had undergone pancreaticoduodenectomy for bile duct cancer. Postoperatively, she had developed grade $\mathrm{C}$ pancreatic fistula diagnosed according to International Study Group of Pancreatic Surgery criteria [2]. Rehabilitation was started after hospital admission, and oral intake was satisfactory. Over the next 8 days, symptoms gradually deteriorated. Level of consciousness and truncal ataxia worsened; bending the lower limbs became impossible, and walking was difficult due to an inability to maintain balance. Blood examinations including complete blood count, serum glucose, blood urea, serum creatinine, and liver function tests were all within normal ranges. Laboratory examinations did not indicate coagulopathy or any infectious diseases. Urinalysis and chest X-ray were also normal. Abdominal CT revealed mild dilatation of the residual stomach, and stenosis of the gastrojejunostomy was suspected. The patient's somnolent tendencies worsened (Glasgow Coma Scale [GCS] score, 9/15) and rapid, shallow breathing developed. Arterial blood gas analysis revealed severe lactic acidosis (Table 1). We suspected that the lactic acidosis was related to thiamine deficiency. Magnetic resonance imaging (MRI) showed bilaterally symmetrical hyperintensities around the third ventricle, cerebral aqueduct, and mammillary bodies on T2-weighted FLAIR (fluid-attenuated inversion recovery) sequences (Fig. 1). 
Based on a suspected diagnosis of WE, intravenous infusion of thiamine at a dosage of $1,200 \mathrm{mg}$ once a day was carried out for 3 days. Blood lactic acid level dramatically improved and normalized within $20 \mathrm{~h}$ of starting thiamine administration. GCS score improved to $15 / 15$, and truncal ataxia resolved. After starting total parenteral nutrition, oral intake also improved. Brain MRI showed normal-intensity signals around the cerebral aqueduct, but atrophic changes were observed in the mammillary bodies, indicating chronic-stage WE (Fig. 2). Thiamine at $400 \mathrm{mg} /$ day was given intravenously for 6 days, followed by parenteral infusion of thiamine at $100 \mathrm{mg} /$ day. Blood thiamine concentration was $2.4 \mu \mathrm{g} / \mathrm{dL}$ (normal range, 2.6-5.8 $\mu \mathrm{g} / \mathrm{dL}$ ) when we suspected WE due to severe lactic acidosis and the findings of brain MRI. The patient was transferred to another hospital for rehabilitation 70 days after hospital admission, with mild memory impairment and gait ataxia.

\section{Discussion and Conclusions}

The present case highlighted two important clinical issues: (1) that WE developed after pancreaticoduodenectomy; and (2) that blood gas analysis was useful for diagnosing WE.

In this case, WE developed 12 months after pancreaticoduodenectomy. The physiopathological mechanisms underlying the occurrence of WE might be related to two factors. First, stenosis of the gastrojejunostomy caused an eating disorder. Oral intake disorder has been reported to induce insufficient thiamine uptake and utilization [3]. In this case, a percutaneous drainage tube was inserted behind the gastrojejunostomy for the pancreatic fistula. Stenosis of the gastrojejunostomy might thus have developed gradually due to residual inflammation and the healing process for pancreatic fistula. Second, the absorption of thiamine is not yet clearly understood, but maximal thiamine absorption is known to occur in the duodenum and, to a lesser extent, the entire small intestine $[4,5]$. Thiamine deficiency might thus develop due to the lack of duodenum after pancreaticoduodenectomy. In the present case, blood gas analysis was useful for diagnosing WE. This pathology is difficult to diagnose clinically because of the lack of abnormalities specific to WE [6]. The clinical triad of mental status changes, oculomotor manifestations, and gait ataxia is not always a sensitive or reliable diagnostic tool [7, 8]. Harper et al. [8] noted that $19 \%$ of WE cases did not present with the classical clinical triad, meaning that a substantial proportion of patients with WE are undiagnosed or misdiagnosed. The present case showed pronounced lactic acidosis with a concurrent disturbance in consciousness. Lactic acidosis is a specific marker of tissue hypoxia [9]. When acidosis is found after gastrointestinal surgery, we generally consider intestinal ischemia, sepsis, diarrhea, and renal insufficiency as possible etiologies. In this case, no specific abnormalities were detected from laboratory examinations, with the exception of acidosis on examination of blood gases, and no specific abnormalities were found on brain or abdominal CT.

As thiamine is essential for mitochondrial metabolism, lactic acidosis in WE results from failure of oxygen utilization due to mitochondrial dysfunction $[10,11]$. Determination of thiamine concentrations in the blood is central to the diagnosis of WE. However, such findings should be used in conjunction with other clinical findings, because the sensitivity and specificity of the test and its reflection of tissue thiamine concentration in the brain have not been established [11]. Furthermore, laboratory measurement of thiamine concentration requires 6-8 days. Brain MRI represents another tool for the diagnosis of WE when clinical diagnosis 
is difficult. However, Antunez et al. [12] reported that the sensitivity and specificity of MRI in the diagnosis of WE were 53 and 93\%, respectively. Because of the nonspecific clinical presentation of WE, lactic acidosis is easily measured via blood gas examination and may be useful for the diagnosis of WE.

WE is usually related to chronic alcoholism with malnutrition. Other causes of WE are hyperemesis gravidarum, parenteral nutrition without thiamine, cancer, and chemotherapeutic treatments $[13,14]$. WE has also been reported after gastrointestinal surgery $[15,16]$. However, it is not well known whether WE can occur after a long period of latency following gastrointestinal surgery associated with minor changes in dietary habits and together with a long-standing latent deficiency of thiamine induced by gastrointestinal conditions [17]. Due to the nonspecific clinical symptoms of WE, delayed diagnosis may lead to irreversible brain damage and death [18]. In the case presented here, prompt thiamine treatment could have resulted in earlier recovery and thus avoided unnecessary prolongation of the hospital stay.

In conclusion, we should recognize that pancreaticoduodenectomy can cause WE and that blood gas analysis can be useful for diagnosing this condition. Further studies are necessary to determine whether WE may be much more prevalent and whether examination of blood gases can contribute to earlier diagnosis of WE.

\section{Statement of Ethics}

This study was approved by the Institutional Review Board of Fukuyama City Hospital (approval \#316). Written informed consent was obtained from the patients for publication of this case report and of the related images. A copy of the written consent is available for review by the Editor-in-Chief of this journal.

\section{Disclosure Statement}

All authors declare that they have no competing interests.

\section{Funding Sources}

The authors did not receive any financial support.

\section{Author Contributions}

K.M. and H.S. conceived the idea for this case report and wrote the manuscript. M.R., H.S., T.U., and K.Y. made substantial contributions to the concept and design of the article and acquisition of materials. M.H., S.O., K.S., and N.T. provided crucial ideas and helped with data interpretation. All authors read and approved the final manuscript. 


\section{Case Reports in Clinical Nutrition}

Case Rep Clin Nutr 2019;2:1-7

DOI: $10.1159 / 000499035$

(C) 2019 The Author(s). Published by S. Karger AG, Base www.karger.com/crc

Monden et al.: Wernicke's Encephalopathy after Pancreaticoduodenectomy: A Case Report

\section{References}

1 Sechi G, Serra A. Wernicke's encephalopathy: new clinical settings and recent advances in diagnosis and management. Lancet Neurol. 2007 May;6(5):442-55.

2 Bassi C, Dervenis C, Butturini G, Fingerhut A, Yeo C, Izbicki J, et al.; International Study Group on Pancreatic Fistula Definition. Postoperative pancreatic fistula: an international study group (ISGPF) definition. Surgery. 2005 Jul;138(1):8-13.

3 Koike H, Misu K, Hattori N, Ito S, Ichimura M, Ito H, et al. Postgastrectomy polyneuropathy with thiamine deficiency. J Neurol Neurosurg Psychiatry. 2001 Sep;71(3):357-62.

4 Reidling JC, Said HM. In vitro and in vivo characterization of the minimal promoter region of the human thiamin transporter SLC19A2. Am J Physiol Cell Physiol. 2003 Sep;285(3):C633-41.

5 Laforenza U, Patrini C, Alvisi C, Faelli A, Licandro A, Rindi G. Thiamine uptake in human intestinal biopsy specimens, including observations from a patient with acute thiamine deficiency. Am J Clin Nutr. 1997 Aug;66(2):320-6.

6 Makarewicz W, Kaska L, Kobiela J, Stefaniak T, Krajewski J, Stankiewicz M, et al. Wernicke’s syndrome after sleeve gastrectomy. Obes Surg. 2007 May;17(5):704-6.

7 Isenberg-Grzeda E, Kutner HE, Nicolson SE. Wernicke-Korsakoff-syndrome: under-recognized and undertreated. Psychosomatics. 2012 Nov-Dec;53(6):507-16.

8 Harper CG, Giles M, Finlay-Jones R. Clinical signs in the Wernicke-Korsakoff complex: a retrospective analysis of 131 cases diagnosed at necropsy. J Neurol Neurosurg Psychiatry. 1986 Apr;49(4):341-5.

9 Demir IE, Ceyhan GO, Friess H. Beyond lactate: is there a role for serum lactate measurement in diagnosing acute mesenteric ischemia? Dig Surg. 2012;29(3):226-35.

10 Donnino MW, Andersen LW, Chase M, Berg KM, Tidswell M, Giberson T, et al.; Center for Resuscitation Science Research Group. Randomized, Double-Blind, Placebo-Controlled Trial of Thiamine as a Metabolic Resuscitator in Septic Shock: A Pilot Study. Crit Care Med. 2016 Feb;44(2):360-7.

11 Donnino MW, Vega J, Miller J, Walsh M. Myths and misconceptions of Wernicke's encephalopathy: what every emergency physician should know. Ann Emerg Med. 2007 Dec;50(6):715-21.

12 Antunez E, Estruch R, Cardenal C, Nicolas JM, Fernandez-Sola J, Urbano-Marquez A. Usefulness of CT and MR imaging in the diagnosis of acute Wernicke's encephalopathy. AJR Am J Roentgenol. 1998 Oct;171(4):11317.

13 Caine D, Halliday GM, Kril JJ, Harper CG. Operational criteria for the classification of chronic alcoholics: identification of Wernicke's encephalopathy. J Neurol Neurosurg Psychiatry. 1997 Jan;62(1):51-60.

14 Turner JE, Alley JG, Sharpless NE. Medical problems in patients with malignancy: case 2. Wernicke's encephalopathy: an unusual acute neurologic complication of lymphoma and its therapy. J Clin Oncol. 2004 Oct;22(19):4020-2.

15 Pardo-Aranda F, Perez-Romero N, Osorio J, Rodriguez-Santiago J, Muñoz E, Puértolas N, et al. Wernicke’s encephalopathy after sleeve gastrectomy: literature review. Int J Surg Case Rep. 2016;20:92-5.

16 Tozzo P, Caenazzo L, Rodriguez D, Bolcato M. Delayed diagnosis of Wernicke encephalopathy with irreversible neural damage after subtotal gastrectomy for gastric cancer: A case of medical liability? Int J Surg Case Rep. 2017;30:76-80.

17 Shimomura T, Mori E, Hirono N, Imamura T, Yamashita H. Development of Wernicke-Korsakoff syndrome after long intervals following gastrectomy. Arch Neurol. 1998 Sep;55(9):1242-5.

18 Busani S, Bonvecchio C, Gaspari A, Malagoli M, Todeschini A, Cautero N, et al. Wernicke's encephalopathy in a malnourished surgical patient: a difficult diagnosis. BMC Res Notes. 2014 Oct;7(1):718. 


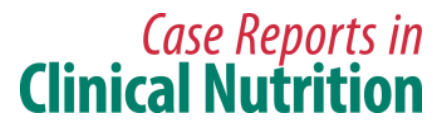

Case Rep Clin Nutr 2019;2:1-7 www.karger.com/crc

Monden et al.: Wernicke's Encephalopathy after Pancreaticoduodenectomy: A Case

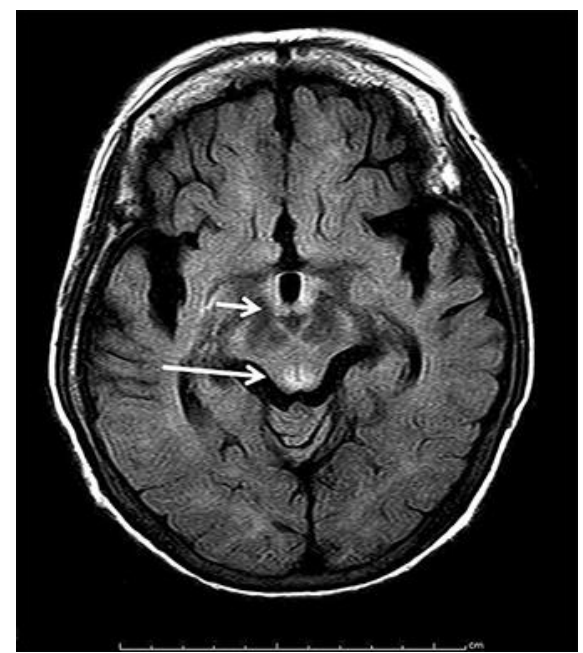

Fig. 1. Magnetic resonance imaging of the brain before initiation of thiamine replacement therapy. T2weighted fluid-attenuated inversion recovery sequence shows bilaterally symmetrical hyperintensities around the cerebral aqueduct (long arrow) and mammillary bodies (short arrow).

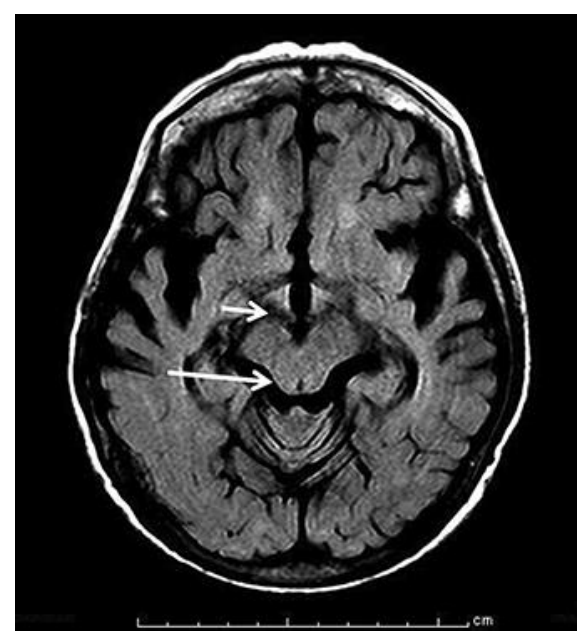

Fig. 2. Magnetic resonance imaging of the brain after thiamine replacement therapy. T2-weighted fluidattenuated inversion recovery sequence shows normal-intensity signals around the cerebral aqueduct (long arrow), and atrophic changes in mammillary bodies (short arrow). 


\section{Case Reports in \\ Clinical Nutrition}

\begin{tabular}{|c|c|}
\hline DOI: 10.1159/000499035 & $\begin{array}{l}\text { (c) } 2019 \text { The Author(s). Published by S. Karger AG, Basel } \\
\text { www.karger.com/crc }\end{array}$ \\
\hline
\end{tabular}

www.karger.com/crc

Monden et al.: Wernicke's Encephalopathy after Pancreaticoduodenectomy: A Case Report

Table 1. Results of arterial blood gas analysis

\begin{tabular}{lcc}
\hline Value & Result & Reference range \\
\hline $\mathrm{pH}$ & 7.347 & 7.350 to 7.450 \\
$\mathrm{pCO}_{2}, \mathrm{~mm} \mathrm{Hg}$ & 25.5 & 32.0 to 45.0 \\
$\mathrm{pO}_{2}, \mathrm{~mm} \mathrm{Hg}$ & 96.9 & 83.0 to 108.0 \\
$\mathrm{HCO}_{3}-\mathrm{mmol} / \mathrm{L}$ & 13.6 & 21.0 to 28.0 \\
$\mathrm{BE}, \mathrm{mmol} / \mathrm{L}$ & -10.9 & -2.0 to 3.0 \\
$\mathrm{Lac}, \mathrm{mmol} / \mathrm{L}$ & 9.1 & 0.5 to 1.6 \\
$\mathrm{AnGap}, \mathrm{mmol} / \mathrm{L}$ & 18.0 & 10.0 to 20.0 \\
$\mathrm{Na}^{+}, \mathrm{mEq} / \mathrm{L}$ & 145 & 136 to 146 \\
$\mathrm{~K}^{+}, \mathrm{mEq} / \mathrm{L}$ & 3.5 & 3.4 to 4.5 \\
$\mathrm{GLU}, \mathrm{mg} / \mathrm{dL}$ & 205 & 70 to 105 \\
\hline
\end{tabular}

AnGap, anion gap; $\mathrm{BE}$, base excess; $\mathrm{GLU}$, glucose; $\mathrm{K}^{+}$, potassium; Lac, lactate; $\mathrm{Na}^{+}$, sodium. 